\title{
Extreme super-resolution using the Spherical Geodesic Waveguide
}

\author{
Juan Carlos Miñano*, Juan Carlos González, Pablo Benítez, Dejan Grabovičkić, \\ Universidad Politécnica de Madrid, Cedint. Campus de Montegancedo 28223 Madrid, Spain
}

\begin{abstract}
Leonhardt demonstrated (2009) that the 2D Maxwell Fish Eye lens (MFE) can focus perfectly 2D Helmholtz waves of arbitrary frequency, i.e., it can transport perfectly an outward (monopole) 2D Helmholtz wave field, generated by a point source, towards a "perfect point drain" located at the corresponding image point. Moreover, a prototype with $\lambda / 5$ superresolution (SR) property for one microwave frequency has been manufactured and tested (Ma et al, 2010). Although this prototype has been loaded with an impedance different from the "perfect point drain", it has shown super-resolution property. However, neither software simulations nor experimental measurements for a broad band of frequencies have yet been reported. Here we present steady state simulations for two cases, using perfect drain as suggested by Leonhardt and without perfect drain as in the prototype. All the simulations have been done using a device equivalent to the MFE, called the Spherical Geodesic Waveguide (SGW). The results show the super-resolution up to $\lambda / 3000$, for the system loaded with the perfect drain, and up to $\lambda / 500$ for a not perfect load. In both cases super-resolution only happens for discrete number of frequencies. Out of these frequencies, the SGW does not show super-resolution in the analysis carried out.
\end{abstract}

Keywords: Super-resolution, Perfect Imaging, Maxwell Fish Eye, Spherical Geodesic Waveguide, Perfect Drain.

\section{INTRODUCTION}

"Super-resolution" stands for the capacity of an optical system to produce images with details below the classic Abbe diffraction limit. In the last decade super-resolution has been shown experimentally with devices made of left-handed materials [1][2] (that is, materials with negative dielectric and magnetic constants [3][4]). Unfortunately, high absorption and small (wavelength scale) source-to-image distance are both present in these experiments. Nevertheless, these devices have been claimed to reach the theoretical limit of infinite resolution [3].

An alternative device for perfect imaging has recently been proposed [6][7]: the Maxwell Fish Eye (MFE) lens. Unlike previous perfect imaging devices, MFE uses materials with a positive, isotropic refractive index distribution. This device is very well known in the framework of Geometrical Optics because it is an Absolute Instrument [5], so every object point has a stigmatic image point.

Leonhardt [6] analyzed Helmholtz wave fields in the MFE lens in two dimensions (2D). These Helmholtz wave fields describe TE-polarized modes in a cylindrical MFE, i.e., modes in which electric field vector points orthogonally to the cross section of the cylinder. Leonhardt found a family of Helmholtz wave fields which have a monopole asymptotic behavior at an object point as well as at its stigmatic image point. Each one of these solutions describes a wave propagating from the object point to the image point. It coincides asymptotically with an outward (monopole) Helmholtz wave at the object point, as generated by a point source, and with an inward (monopole) wave at the image point, as it was sunk by an "infinitely-well localized drain" (which we call a "perfect point drain"). This perfect point drain absorbs the incident wave, with no reflection or scattering. This result has also been confirmed via a different approach [8].

The physical significance of a passive perfect point drain has been controversial [9]-[18]. In references [6] and [5] the perfect point drain was not physically described, but only considered as a mathematical entity (a point drain is represented by Dirac-delta as the point source). However, a rigorous example of a passive perfect point drain for the MFE has recently been found, clarifying the controversy [19]. It consists of a dissipative region whose diameter tends towards zero and whose complex permittivity $\varepsilon$ takes a specific value depending on the operation frequency. 
Two sets of experiments have recently been carried out to support the super-resolution capability in the MFE. In the first one, super-resolution with positive refraction has been demonstrated for the very first time at a microwave-frequency $(\lambda=3 \mathrm{~cm})[20][21]$. The experimental results showed that two sources with a distance of $\lambda / 5$ from each other (where $\lambda$ denotes the local wavelength $\lambda=\lambda_{0} / \mathrm{n}$ ) could be resolved with an array made up of 10 drains spaced $\lambda / 20$, which exceeded the $\sim \lambda / 2.5$ classic diffraction limit. Results with closer sources were not reported, but it should be noted that this experiment was limited to the resolution of the array of drains.

The second set of experiments has been carried out for the near infrared frequency $(\lambda=1.55 \mu \mathrm{m})$, but resolution below the diffraction limit was not found [22]. The authors assume that the failure in the experimental demonstration is due to manufacturing flaws in the prototype.

Although the perfect drain has not been used in these experiments, i.e. there was a reflected wave from the drain to the source, the MFE has shown super-resolution for microwave frequencies. This means that the perfect drain is not necessary for reaching the super-resolution (see also [23]). However, in this paper we will show that the use of the perfect drain increases super-resolution.

Recently, a device equivalent to the MFE, Spherical Geodesic Waveguide (SGW) made for microwave frequencies has been presented [23][24]. The SGW is a spherical waveguide filled with a non-magnetic material and isotropic refractive index distribution proportional to $1 / r\left(\varepsilon=\left(r_{0} / r\right)^{2}\right.$ and $\left.\mu=1\right), r$ being the distance to the center of the spheres. Transformation Optics theory [25] proves that the TE-polarized electric modes of the cylindrical MFE are transformed into radial-polarized modes in the SGW, so both have the same imaging properties. When the waveguide thickness is small enough, the variation of the refractive index within the two spherical shells can be ignored resulting in a constant refractive index within the waveguide. In [23] the SGW has been analyzed using two coaxial probes (source and drain) loaded with the characteristic impedances. The results have shown the super-resolution up to $\lambda / 500$ for a discrete number of frequencies, called notch frequencies, that are close to the well known Schumman Resonance frequencies of spherical systems. For other frequencies the system did not present resolution below diffraction limit. In [23] the perfect drain has not been used, thus beside the incident wave, a reflected wave existed in the SGW as well. However, the superresolution properties have been shown.

Herein, we present an improvement of the super-resolution using the SGW with the perfect drain. The perfect drain is realized using a circuit (made of a resistance and a capacitor) connected to the drain coaxial probe. The difference between the presented drain and the perfect drain proposed in [19] is the practical realization. While in [19] the perfect drain is made of a material with complex permittivity $\varepsilon$, here it is only a coaxial line loaded with a resistor and a capacitor of conventional values (for example, $R=2.57 \Omega$ and $C=55.05 \mathrm{pF}$ for $f=0.25 \mathrm{GHz}$ ). Using the circuital model for the perfect drain, the Comsol simulations have shown the super-resolution up to $\lambda / 3000$ for the same discrete frequencies as in [23], which is much higher than the $\lambda / 500$ obtained without perfect drain.

\section{ANALYZED STRUCTURE}

\subsection{Maxwell Fish Eye lens and Spherical Geodesic Waveguide}

A MFE is a lens with the following refraction index distribution:

$$
n(\rho)=\frac{2 n_{0}}{1+(\rho / a)^{2}}
$$

where $\rho$ is the distance to the origin, which in the $2 \mathrm{D}$ case is $\rho^{2}=x^{2}+y^{2}$. Within the Geometrical Optics framework, the rays emitted from an arbitrary object point $\left(x_{0}, 0\right)$ will be stigmatically imaged onto its image point $\left(-a^{2} / x_{0}, 0\right)$. The wave propagating from an object point $\left(x_{0}, 0\right)$ onto its image point $\left(-a^{2} / x_{0}, 0\right)$ found by Leonhardt [6] (which is known as forward running-wave Legendre function [27]), is given by: 


$$
\begin{aligned}
& \mathbf{E}(x, y, z)=E_{z}(x, y) \mathbf{z}=A\left(P_{v}(\zeta)+i \frac{2}{\pi} Q_{v}(\zeta)\right) \mathbf{z} \\
& \left(\frac{a}{n_{o}} k_{0}\right)^{2}=v(v+1) \quad k_{0}=2 \pi f \sqrt{\mu_{0} \varepsilon_{0}} \quad \zeta=\frac{\left|z^{\prime}\right|^{2}-a^{2}}{\left|z^{\prime}\right|^{2}+a^{2}} \quad z^{\prime}=\frac{z-x_{0}}{z \frac{x_{0}}{a^{2}}+1} \quad z=x+i y
\end{aligned}
$$

Here $A$ is a complex constant, and $P_{v}, Q_{v}$ are the Legendre functions [28]. The field $E_{z}$ diverges at the points $\left(x_{\theta}, 0\right)$ and $\left(-a^{2} / x_{0}, 0\right)$, i.e., at both the source (located at the object point) and the drain (located at the image point). Besides the point source, this solution requires a perfect point drain which is a theoretical concept that can be modeled as an infinitely small region centered around the image point and with a particular complex permittivity distribution [19].

Using Transformation Optics it was proven that the fields given by Eq. (2) in a 2D MFE are transformed into radial fields in the SGW filled with a refractive index medium with law $n(r)=a n_{0} / r$ (where $r^{2}=x^{2}+y^{2}+z^{2}$ ). The radial field $\mathbf{E}(r, \theta, \phi)=E_{r}(r, \theta, \phi) \mathbf{r}$ in the SGW is related to the MFE field (Eq. (2)) of the corresponding point by:

$$
E_{r}(r, \theta, \phi)=E_{z}(\zeta) \quad \zeta=\cos \theta
$$

Corresponding points in the MFE and SGW are related by a stereographic projection. Source and drain points $\left(x_{0}, 0\right)$ and $\left(-a^{2} / x_{0}, 0\right)$ are transformed into opposite poles of the SGW. The forward running-wave Legendre function is transformed into a wave with rotational symmetry with respect to the line passing through object and image points. The perfect point drain complex permittivity distribution is transformed into another one.

When the drain of the SGW is not perfect but still rotationally symmetric, the field can be expressed as follows[19]:

$$
E_{r}(r, \theta, \phi)=E_{r}(\zeta)=A F_{v}(\zeta)+B R_{v}(\zeta)
$$

where

$$
F_{v}(\zeta)=P_{v}(\zeta)+i \frac{2}{\pi} Q_{v}(\zeta) \quad R_{v}(\zeta)=P_{v}(\zeta)-i \frac{2}{\pi} Q_{v}(\zeta)
$$

$F_{v}$ is forward running-wave Legendre function and $R_{v}$ is the reverse one [27]. $\zeta$ is given in Eq. (3).

\subsection{Microwave circuit and parameters of the simulation.}

The SGW is bounded by two metallic spherical shells. The media between the shells is air. Two coaxial probes have been used to simulate the source and drain in the SGW. Consider the microwave circuit consists of the generator $V_{g}$ with the impedance $Z_{g}$ (on the source port side), coaxial lines, the SGW, and the load with the impedance $Z_{L}$ (on the drain port side), as shown in Figure 1 with:

$R_{M}=1005 \mathrm{~mm}, R_{m}=1000 \mathrm{~mm}, a=5 \mathrm{~mm}$ (Diameter of the inner conductor of the coaxial), $b=10 \mathrm{~mm}$ (Diameter of the outer conductor of the coaxial), $L=20 \mathrm{~mm}$.

The same circuit has been analyzed in [23] with the condition $Z_{L}=Z_{g}=Z_{0}$, where $Z_{0}$ is the characteristics impedance of the coaxial line. In this case:

$$
\left|S_{21}\right|^{2}=\frac{P_{\text {load }}}{P_{\max }}
$$

Where, $S_{21}$ is the scattering parameter of the circuit [26], $P_{\text {load }}$ is the power delivered to the load $Z_{L}$ and $P_{\max }$ is the maximum power that can be delivered by the generator. In [23] the results have been presented using function $\left|S_{21}\right|^{2}$ obtained for different frequencies and displacements $\Delta \theta$.

Here, it is presented also the same analysis using the impedance that simulates the perfect drain (called $Z_{p d}$ ). The procedure to calculate this impedance as a function of frequency is developed in [29]. The SGW with the perfect drain is designed and analyzed in Comsol (Figure 2). In order to show super-resolution properties of the SGW, we have made several simulations for different displacements of the drain port, and for different values of frequency. Special care has been taken to define the mesh of the system. In order to mesh the guide properly, the geometry has been divided into few domains. Each domain is meshed separately according to its geometric and physical properties. Since the guide thickness 
is very low $\left(R_{M}-R_{m}\right) / R_{m}<<1$, the SGW is meshed using a swept mesh (2D triangular mesh from the outer surface is swept to the inner surface, see Figure 2 centre). On the other side, the coaxial cables are meshed with higher density using $3 \mathrm{D}$ tetrahedra. The mesh density is increased since the change of the electric field is significant in the neighbourhood of the coaxial cables.

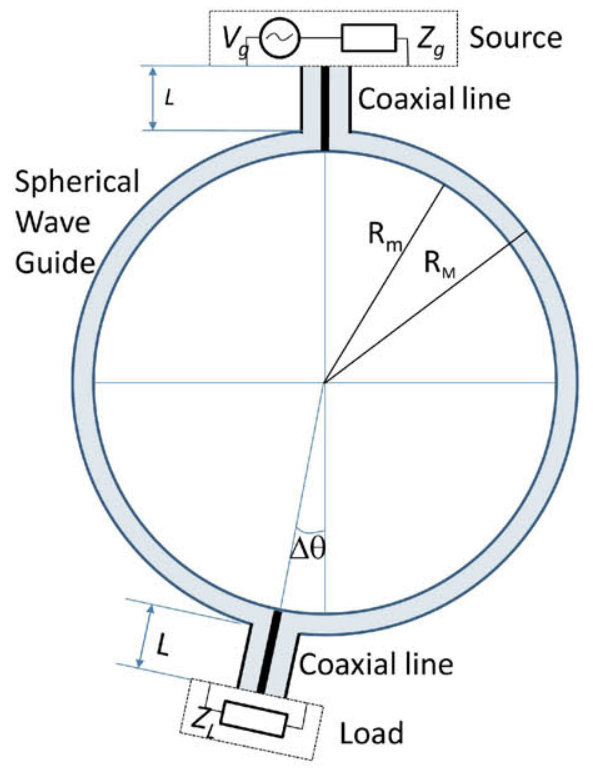

Figure 1. Complete microwave circuit analyzed in this paper formed by: the source $\left(V_{g}\right.$ and $\left.Z_{g}\right)$ connected to a coaxial transmission line of length $L$, the load connected $\left(Z_{L}\right)$ to other identical transmission line and the spherical waveguide. $R_{M}$ and $R_{m}$ are radius of the external and internal metallic spheres
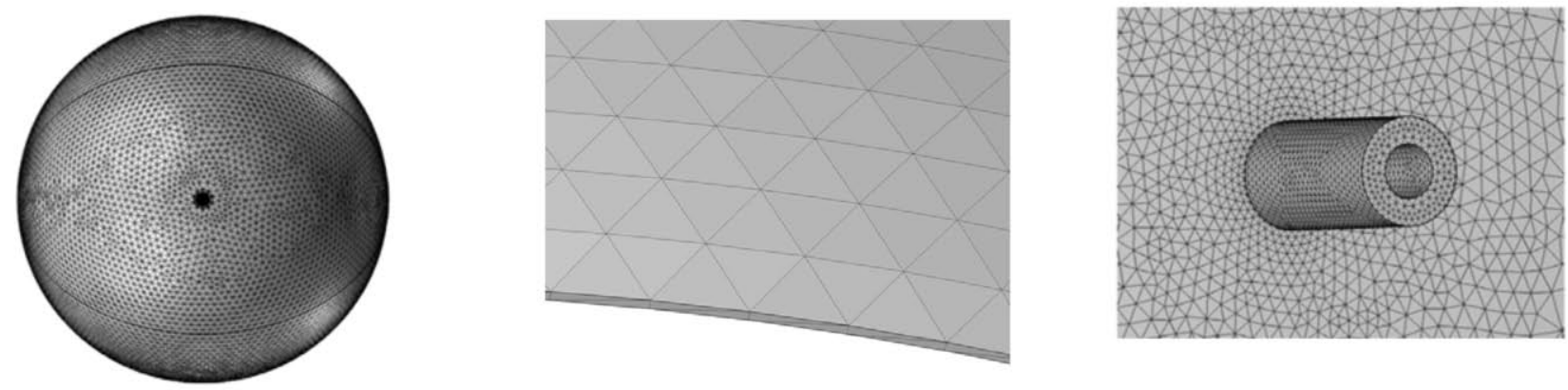

Figure 2. Mesh structure. (left) SGW with coaxial cables, (centre) close up of a piece of spherical shells, (right) close up of one coaxial cable from outside the sphere

\section{RESULTS OF THE SIMULATIONS}

\subsection{SGW loaded with impedance different from the perfect drain.}

Figure 3 shows $\left|S_{21}\right|^{2}$ for a frequency range between $0.2 \mathrm{GHz}$ and $0.4 \mathrm{GHz}$ when the drain port is placed at the source's image point, that is, $\theta=0$ for the source port and $\theta=\pi$ for the drain port. There are peaks of $\left|S_{21}\right|^{2}$ indicating total transmission from the source port towards the drain port. These peaks occur at the so called Schumann resonance frequencies of the spherical systems (see for instance page 374 in [30] ), which correspond to integer values of $v$ in Eq.(2). These peaks are characteristics of resonators as the Fabry-Pérot one (see for instance[31]).

Figure 4 shows $\left|S_{21}\right|^{2}$ when the drain port is shifted $\lambda / 30$ (for $\lambda=1 \mathrm{~m}$ corresponding to $0.3 \mathrm{GHz}$ ) away of source port 
antipode. Although the results are extremely similar, narrow notches in the transmission very close to the Schumann frequencies occur. These notches widen when the drain port is shifted further from the image point of the source, but the null of $\left|S_{21}\right|^{2}$ remains fixed, as can be seen better in Figure 4. The frequencies corresponding to these nulls will be called here notch frequencies.

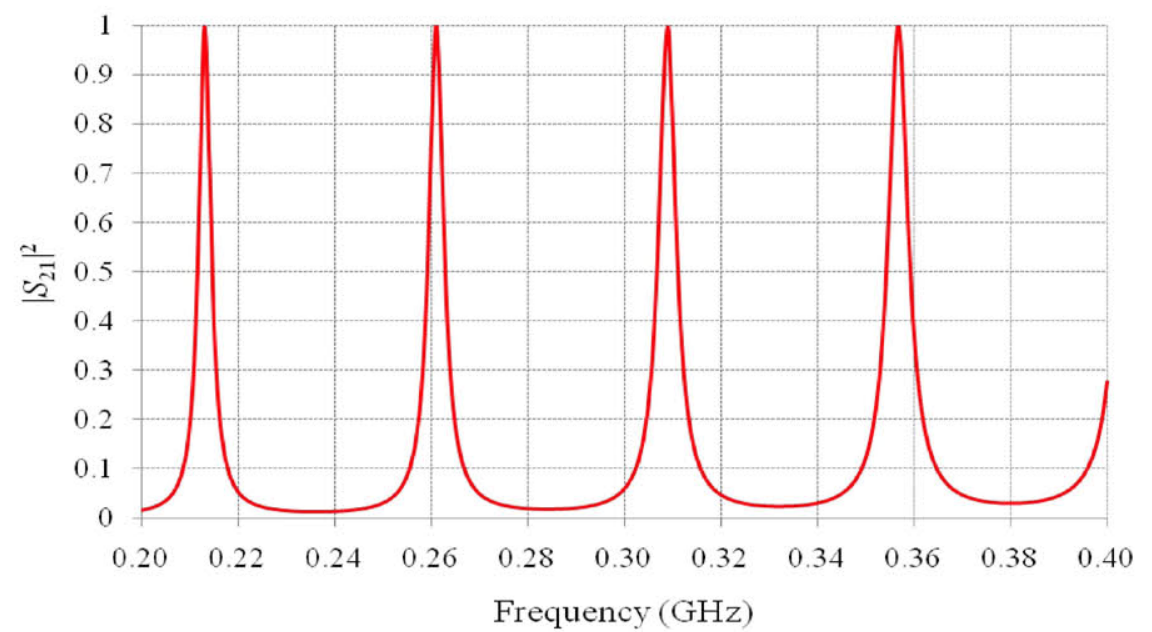

Figure $3\left|S_{21}\right|^{2}$ as function of frequency when the drain and source port's are at opposite poles. The peaks occur at the Schumann resonance frequencies.

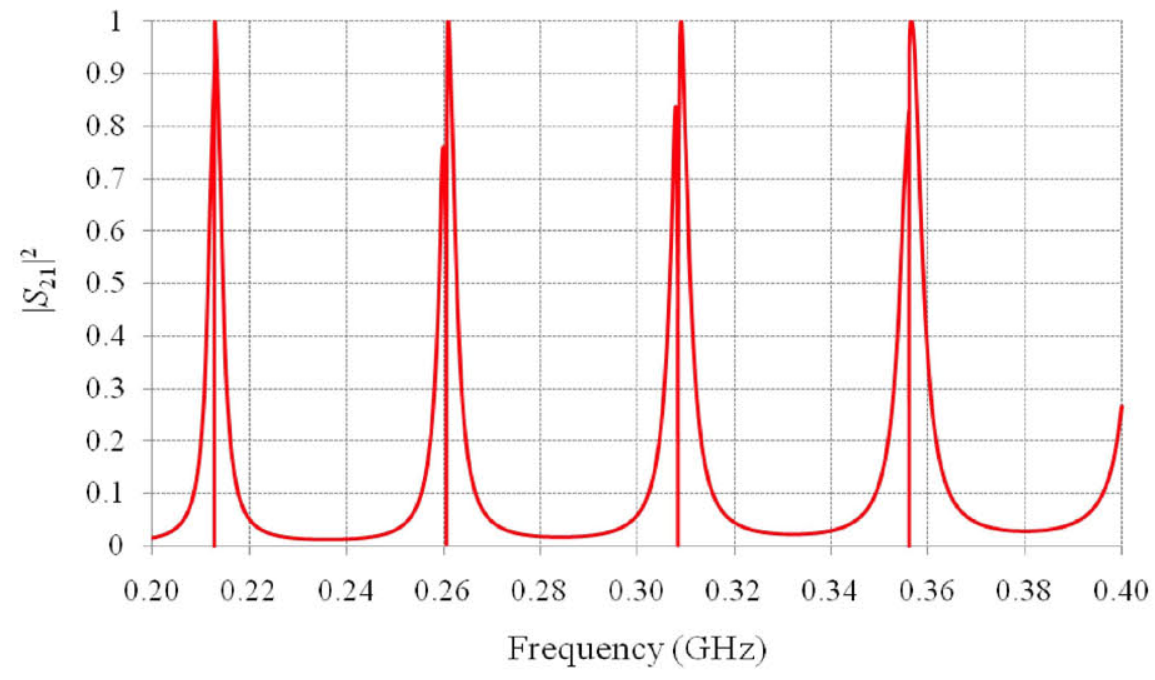

Figure 4. $\left|S_{21}\right|^{2}$ vs frequency when the drain port is shifted $\lambda / 30(\lambda=1 \mathrm{~m})$ from the source port antipode. The results are similar to those presented in Figure 3 in accordance with the classic prediction, except for the very narrow notches near the Schumann frequencies.

Figure 5 shows $\left|S_{21}\right|^{2}$ for different drain port positions in a very narrow band in the neighborhood of the notch frequency corresponding to the second peak in Figure 4 (for which $v=5$ ). The label of each curve indicates the distance between the center of the drain port and the source port antipode. The black curve corresponds to the drain port placed in the source port antipode (it looks flat because of the high zoom in the frequency axis). The other curves correspond to different shifts of the drain port. The shifts are in all cases much smaller than wavelength (from $\lambda / 33$ to $\lambda / 500$ with $\lambda=1.15084047 \mathrm{~m}$ that correspond to $f=0.2606873 \mathrm{GHz}$, see Figure 5). These results are quite surprising, since close to a specific frequency the power transmitted to the drain port suddenly reduces to a value near zero. 


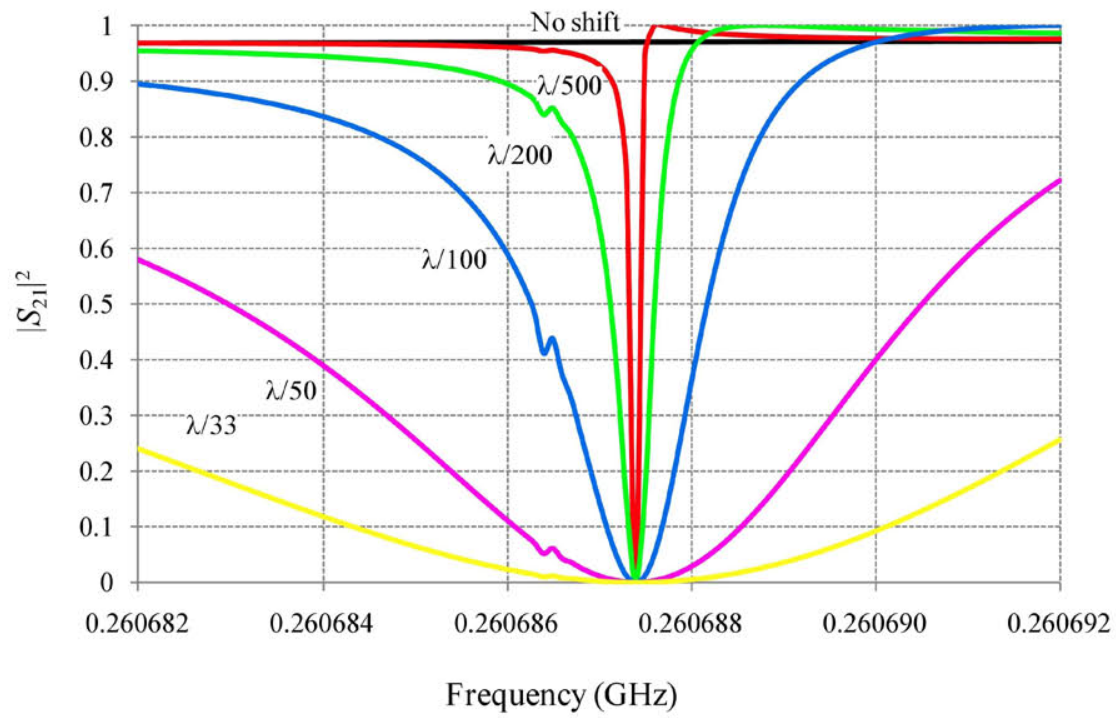

Figure 5. Detail of $\left|\mathrm{S}_{21}\right|^{2}$ as function of the frequency in a narrow band around a notch frequency for different drain port positions (the corresponding shift on the inner sphere of the SGW between the center of the drain port and the source port antipode has been used for labeling). There is no notch in the transmission when the drain port is at the source port antipode (no shift). The notch frequency is $\mathrm{f}=0.2606873 \mathrm{GHz}(\mathrm{v}=4.996)$. The nearest Schumann frequency is $\mathrm{f}=0.26086609 \mathrm{GHz}$ $(v=5)$ which is out the range of this Figure.

Figure 6 shows $\left|S_{21}\right|^{2}$ versus the drain port shift for two frequencies, normalized to value of $\left|S_{21}\right|^{2}$ when the drain port is at antipode of the source (see Figure 3). The blue curve corresponds to $f=0.2847 \mathrm{GHz},(v=5.5)$ i.e., far from a notch frequency.

Let us define "resolution" as the arc length (in wavelength units) that a drain port needs to be shifted so $\left|S_{21}\right|^{2}$ drops to $10 \%$ (not far from the Rayleigh criteria in Optics, which refers to the first null). With this definition, the diffraction limited resolution given by the blue curve is $\lambda / 3.45$. The red curve corresponds to notch frequency $f=0.26068741 \mathrm{GHz}$ $(\nu=4.996)$ which clearly shows a much better resolution.

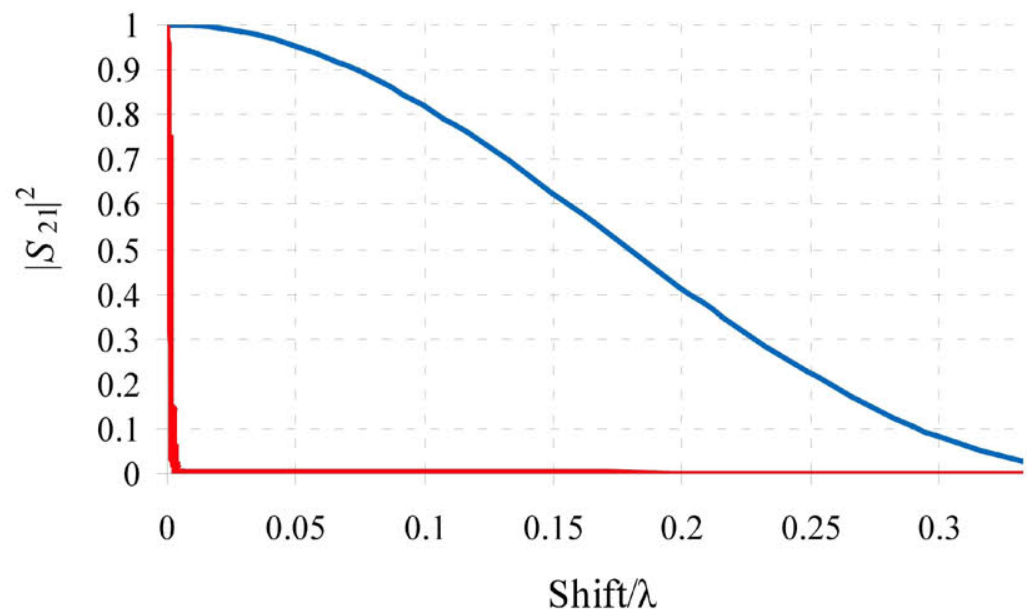

Figure 6. $\left|S_{21}\right|^{2}$ as function of the drain port shift for a frequency near a notch one (red curve) and for a frequency far from a notch one (blue curve).

Figure 7 is a blow-up of Figure 6 in the upper neighborhood of a notch frequency. The graph for frequencies slightly below the notch frequency is similar. Note that Figure 7 shows the same information as Figure 5 but plotting $\left|S_{21}\right|^{2}$ vs. the drain port shift (expressed in units of $\lambda$ ) and using the frequency as a parameter. 


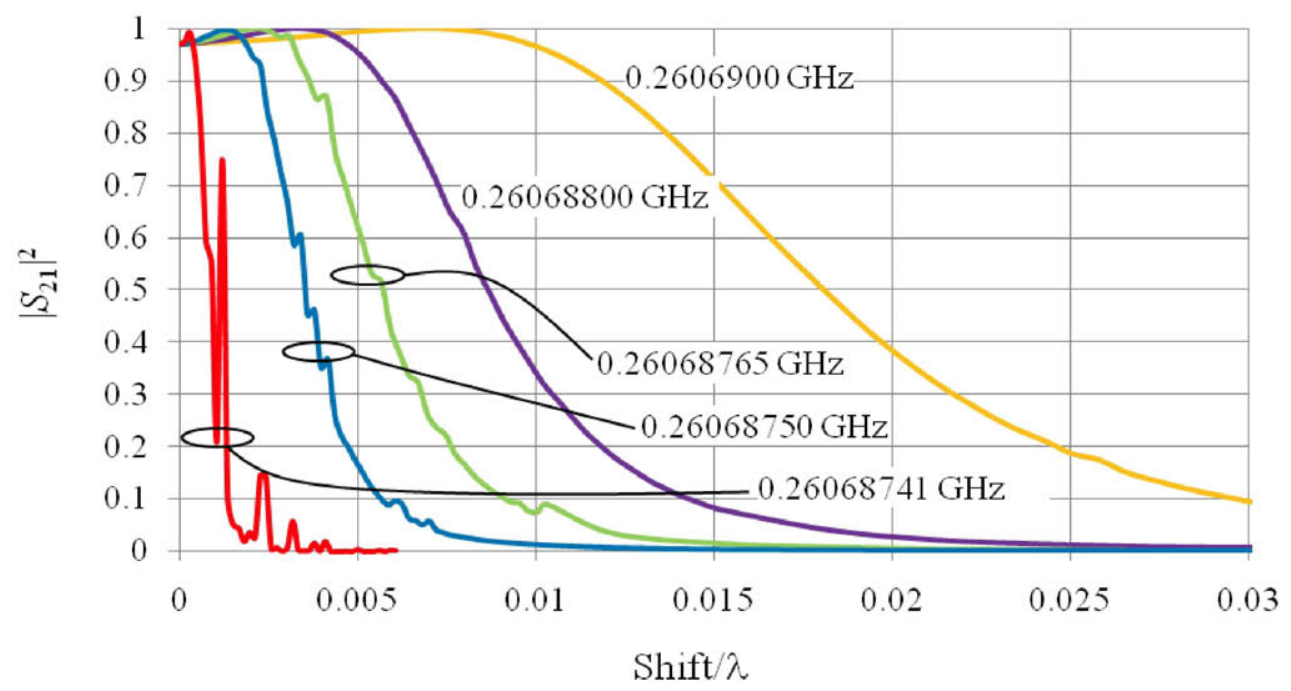

Figure 7. $\left|\mathrm{S}_{21}\right|^{2}$ as function of the drain port shift for different frequencies that present super-resolution of between $\lambda / 30$ and N500.

From the orange to the red curve, increasing resolutions are achieved: $0.03 \lambda$ (that is, $\lambda / 33$ ) for the orange to $\lambda / 500$ for the red. The latter, whose frequency $f=0.26068741 \mathrm{GHz}$ corresponds to $v=4.99636)$ is the highest resolution that we have obtained. Computations for frequencies near the notch frequency show essentially null $\left|\mathrm{S}_{21}\right|^{2}$ values for shifts $>\lambda / 500$ (as in the red line in the picture). $\left|S_{21}\right|^{2}$ values for shifts below $\lambda / 500$ (excepting no shift or shifts very near to zero) and frequencies near a notch frequency are inconsistent (the solver did not converge to a single solution due to numerical errors). It seems that Leonhardt's assertion of infinite resolution (i.e., perfect imaging) may occur for the discrete notch frequencies in the SGW, although the mentioned inconsistencies have prevented us from numerically predicting resolutions beyond $\lambda / 500$. The $\lambda / 500$ resolution is achieved only for a narrow bandwidth $(\approx 20 \mathrm{~Hz}$, which is much smaller than the notch frequency $\approx 0.3 \mathrm{GHz}$ ). If larger bandwidths are needed, lower resolutions (but still sub-wavelength) may be achieved.

Figure 8 shows the bandwidth vs. $N$, meaning that the resolution is better that $\lambda / N$. The bandwidth has been calculated as $f_{\max }-f_{\min }$ with $f_{\max }$ and $f_{\min }$ fulfilling $\left|S_{21}\left(f_{\max }\right)\right|^{2}=\left|S_{21}\left(f_{\min }\right)\right|^{2}=0.1$, using the information of the curves in Figure 7 and similar curves. The linear dependence shown in (slope -2) reveals that the product $N^{2} \times$ bandwidth is constant in the range analyzed here.

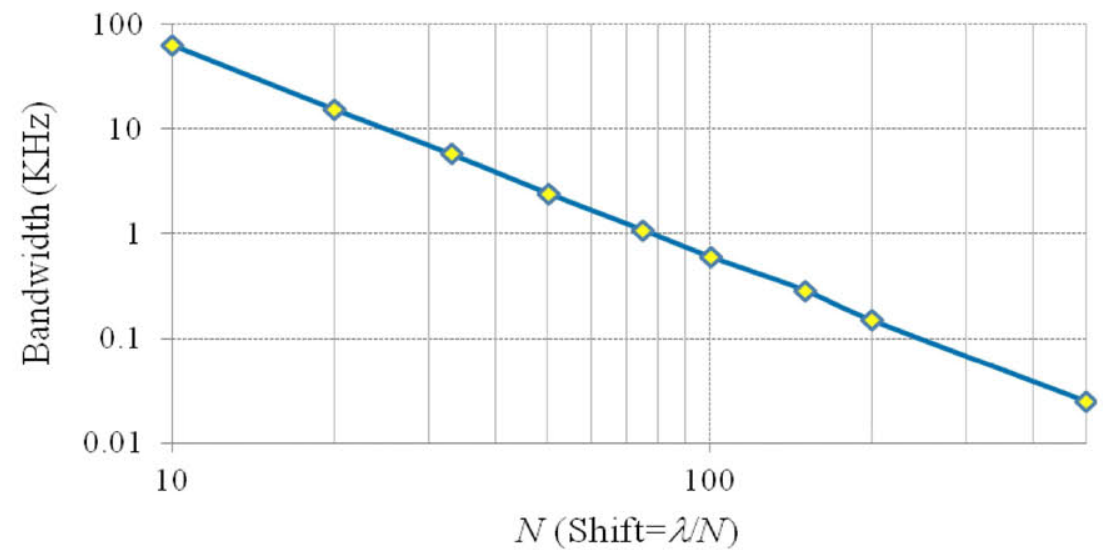

Figure 8. Bandwidth as a function of the resolution. The abscissa axis shows $\mathrm{N}$, meaning that the resolution is better than $\lambda / \mathrm{N}$. 


\subsection{SGW loaded with the perfect drain}

Figure 9 shows $\mathrm{P}_{\text {load }} / \mathrm{P}_{\max }$ as function of the frequency in a narrow band around the same notch frequency of Figure 5. Note that the range of frequencies is the same in both figures, however the maximum resolution has increased from $\lambda / 500$ in Figure 5 to $\lambda / 3000$ in Figure 9.

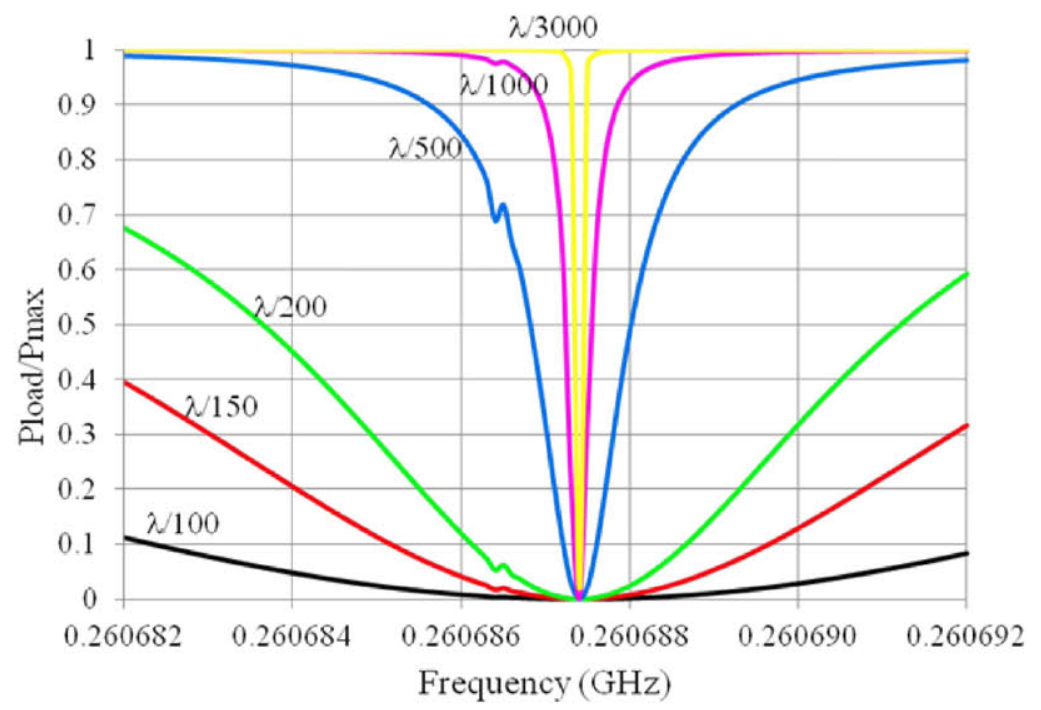

Figure 9. $P_{\text {load }} / P_{\max }$ as function of the frequency in a narrow band around a notch frequency for different drain port positions.

Figure 10 shows $P_{\text {load }} / P_{\max }$ as function of the drain port shifts for different frequencies in the upper neighborhood of a notch frequency. Note that the frequencies are the same as in Figure 7 but the axis of displacement ( $x$ axis) has different scale.

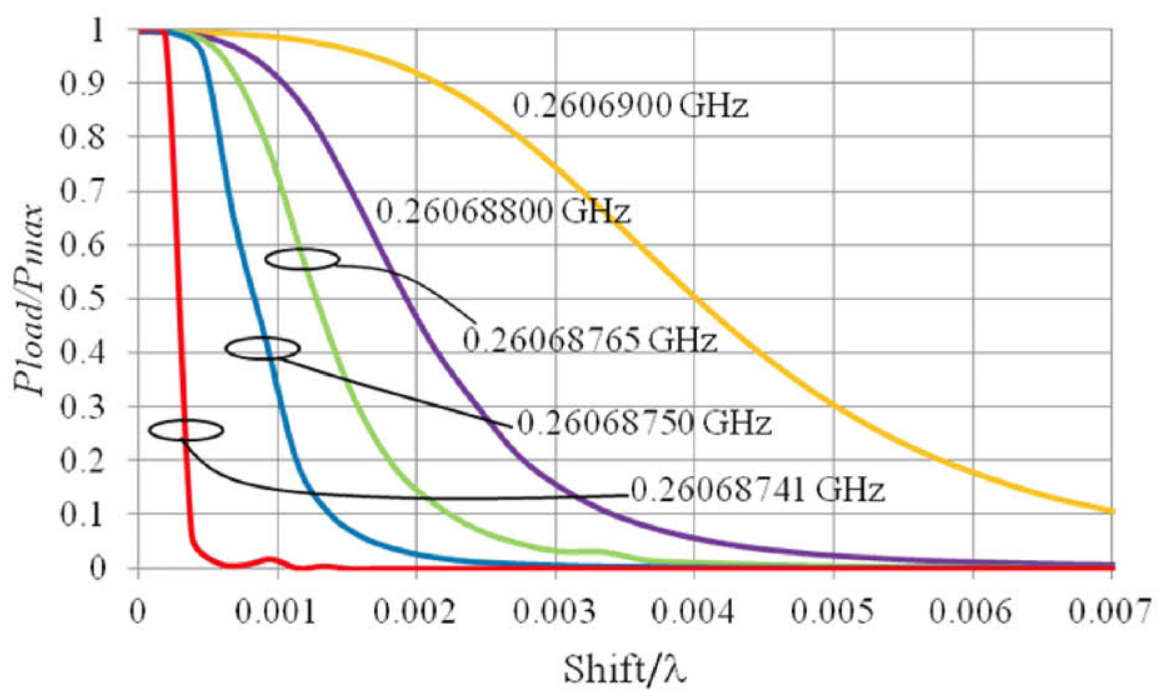

Figure 10. $\mathrm{P}_{\text {load }} / \mathrm{P}_{\max }$ as function of the drain port shift for different frequencies corresponding to super-resolution between $\lambda / 3000$ and $\lambda / 140$.

Figure 11 shows the bandwidth vs. $N$, meaning that the resolution is better that $\lambda / N$. The band-width obtained with the perfect drain is about 20 times higher, which as can be seen comparing Figure 8 and Figure 11. 


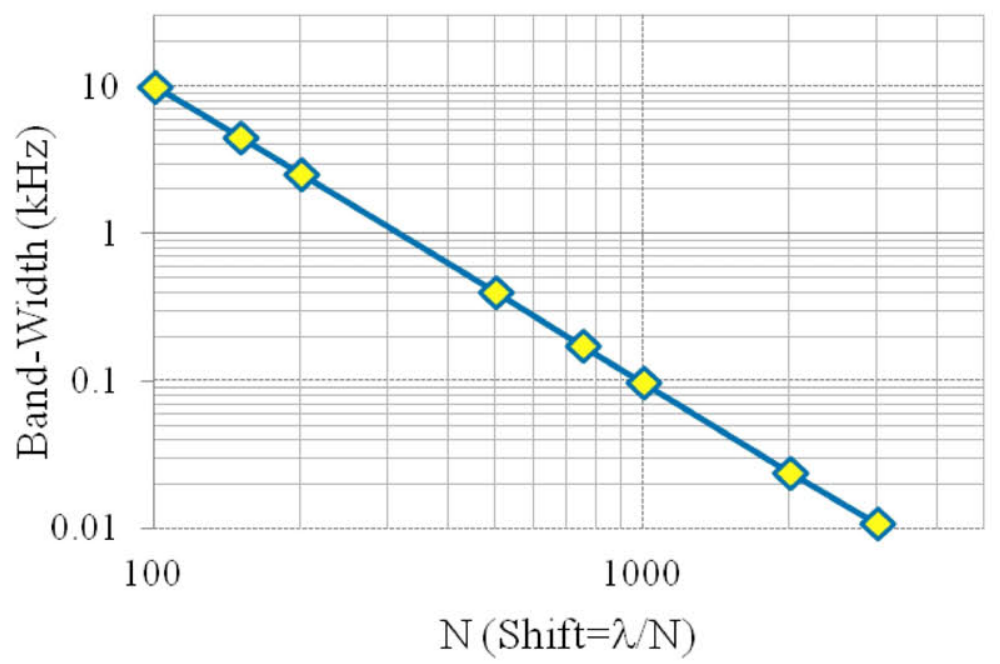

Figure 11. Bandwidth as a function of the resolution. The $\mathrm{x}$ axis shows $N$, meaning that the resolution is better than $\lambda / N$.

\section{CONCLUSIONS}

Leonhardt in [6] and [7] suggested that MFE should produce perfect imaging for any frequency using perfect drains. However, the experiments in [20][21] and simulations from [23] have shown super-resolution properties of the MFE, although the perfect drain has not been used. In these references, the coaxial probes were loaded with their characteristic impedances, so the absorption of the incident wave was not perfect. Leonhardt assumed that the ability of the MFE to propagate the wave, generated by a point source, toward to a perfect point drain was enough to guarantee perfect imaging. This does not seem to be sufficient, since it does not provide information on how much power the drain will absorb when it is displaced out of the image point. The simulations presented here show that super-resolution only occurs for a particular set of frequencies known as notch frequencies. This is the same for both systems, and does not depend on the load at the drain port. The presented results have shown maximum super-resolution $\lambda / 3000$ for the system loaded with the perfect drain, and $\lambda / 500$ for the system loaded with characteristic impedance. Also, using the perfect drain the frequency bandwidth increases 20 times, e.g. for $\lambda / 500$ the bandwidth is about $400 \mathrm{~Hz}$ (for the perfect drain) while it is only $20 \mathrm{~Hz}$ for characteristics impedance (Figure 8 and Figure 11).

\section{ACKNOWLEDGEMENTS}

The authors would like to thank the Spanish Ministry MICINN (ENGINEERING METAMATERIALS: CSD200800066, DEFFIO: TEC2008-03773, SIGMASOLES: PSS-440000-2009-30, PERIMAGE: TEC2011-24019, TEC201016948, INNPACTO SIGMAMODULOS: INN110935C37B), MITYC (ECOLUX: TSI-020100-2010-1131, SEM: TSI020302-2010-65), the Madrid Regional Government (SPIR: 50/2010O.23/12/09,TIC2010 and O-PRO: PIE/209/2010) and UPM (Q090935C59) for the support given in the preparation of the present work.

\section{REFERENCES}

[1] Fang, N., Lee, H., Sun, C. and Zhang, X., "Sub-Diffraction-Limited Optical Imaging with a Silver Superlens," Science 308, 534-537 (2005).

[2] Mesa, F., Freire, F., Marqués, R. and Baena, J. D., "Three dimensional superresolution in material slab lenses: Experiment and theory," Phy. Review B 72, 235117 (2005).

[3] Pendry, J. B., "Negative Refraction makes a Perfect Lens," Phy. Review Let. 85, 3966-3989 (2000). 
[4] Shelby, R. A., Smith, D. R. and Schultz, S., "Experimental verification of negative index of refraction," Science 292, 79 (2001).

[5] Born, M. and Wolf E., [Principles of Optics], Cambridge University Press. Sixth Edition, 143 (1997).

[6] Leonhardt, U., "Perfect imaging without negative refraction," New J. Phys. 11, 093040 (2009).

[7] Leonhardt, U. and Philbin, T. G., "Perfect imaging with positive refraction in three dimensions," Phys. Rev. A 81, 011804 (2010).

[8] Benítez, P., Miñano, J .C. and González, J. C., "Perfect focusing of scalar wave fields in three dimensions," Optics Express 18, 7650-7663 (2010).

[9] Blaikei, R.J., "Comment on 'Perfect imaging without negative refraction," New Journal of Physics 12 (5), $058001(2010)$.

[10] Leonhardt, U., "Reply to comment on 'Perfect imaging without negative refraction," New Journal of Physics. $12(5), 058002(2010)$.

[11] Guenneau, S., Diatta, A. and McPhedran, R. C., "Focusing: coming to the point in metamaterials," Journal of Modern Optics. 57(7), 511-527 (2010).

[12] Merlin, R., "Comment on "Perfect imaging with positive refraction in three dimensions", " Physical Review A. $82(5), 057801(2010)$.

[13] Sun, F. and He, S., "Can Maxwell's fish eye lens really give perfect imaging?," Progress In Electromagnetics Research 108, 307-322 (2010).

[14] Sun, F., Ge, X., C., and He, S., "Can Maxwell's fish eye lens really give perfect imaging? part II. the case with passive drains," Progress In Electromagnetics Research 110, 313-328 (2010).

[15] Leonhardt, U. and Sahebdivan, S., "Perfect imaging: they do not do it with mirrors," Journal of Optics. 13(2), $024016(2010)$.

[16] Leonhardt, U. and Philbin, T.G., "Reply to "Comment on 'Perfect imaging with positive refraction in three dimensions"," Physical Review A. 82(5), 057802 (2010).

[17] Kinsler, P. and Favaro, A., 2010 "Comment on: Reply to comment on 'Perfect imaging without negative refraction'," New J. Phys. 13, 028001 (2010).

[18] Leonhardt, U., "Reply to Comment on Reply to Comment on 'Perfect imaging without negative refraction,"' New J. Phys. 13, 028002 (2011).

[19] González, J. C., Benítez, P. and Miñano, J.C., "Perfect drain for the Maxwell Fish Eye lens," New Journal of Physics 13, 023038 (2011).

[20] Ma, Y.G., Ong, C.K., Sahebdivan, S., Tyc, T. and Leonhardt, U., "Perfect imaging without negative refraction for microwaves," ArXiv: 1007.2530v1, (2010).

[21] Ma, Y.G., Sahebdivan, S., Ong, C.K., Tyc, T. and Leonhardt,U., "Evidence for subwavelength imaging with positive refraction," New J. Phys. 13, 033016 (2010).

[22] Gabrielli, L.H., Leonhardt, U. and Lipson, M., "Perfect imaging in the optical domain using dielectric materials," ArXiv: 1007.2564v1, (2010).

[23] Miñano, J.C., Marqués, R., González, J.C., Benítez, P., Delgado, V., Grabovickic, D. and Freire, M., "SuperResolution for a point source better than $\lambda / 500$ using positive refraction," New Journal of Physics 13,125009 (2011).

[24] Miñano J.C., Benítez P, González JC "Perfect imaging with geodesic waveguides," New Journal of Physics. 12, $123023(2010)$.

[25] Leonhardt, U.,[Geometry and Light: The Science of Invisibility], Dover, (2011).

[26] Pozar, D. M., [Microwave Engineering], John Wiley\&Son Inc, (2005).

[27] Nussenzveig, H. M., "High-frequency scattering by an impenetrable sphere," Ann. Phys. 34, 23 (1965).

[28] Erdélyi, A., Magnus, W., Oberhettinger, F. and Tricomi, F.G., [Higher Transcendental Functions vol I], New York: McGraw-Hill, (1953).

[29] González, J.C., Grabovickic, D., Miñano, J. C. and Benítez P., "Circuital model for the Maxwell Fish Eye perfect drain,". arXiv:1203.2424v1 [physics.optics], (2012).

[30] Jackson, D. J., [Classical Electrodynamics], John Wiley \& Sons, Inc. $3^{\text {rd }}$ ed., (1998).

[31] Solimeno, S., Crosignani, B. and DiPorto, P., [Guiding, Diffraction and Confinement of Optical Radiation], Academic Press, (1986). 